Review

\title{
Traditional Chinese medicine prescription Guizhi Fuling Pills in the treatment of endometriosis
}

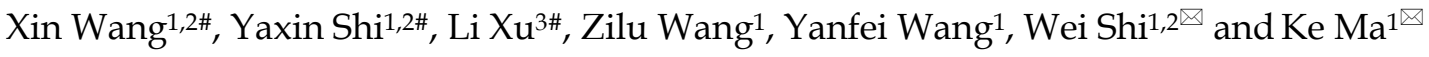 \\ 1. College of Traditional Chinese Medicine, Shandong University of Traditional Chinese Medicine, Jinan 250355, PR China. \\ 2. Department of Gynecology, Affiliated Hospital of Shandong University of Traditional Chinese Medicine, Jinan 250014, PR China. \\ 3. Provincial Hospital Affiliated to Shandong First Medical University, Jinan 250012, PR China. \\ \#Co-first authors with equal contributions to this work.
}

$\triangle$ Corresponding authors: Dr Wei Shi, Department of Gynecology, Affiliated Hospital of Shandong University of Traditional Chinese Medicine, No 16369, Jingshi Road, Jinan, 250014, PR China. E-mail: sw19781214@163.com; Or Dr Ke Ma, College of Traditional Chinese Medicine, Shandong University of Traditional Chinese Medicine, No. 4655, University Road, Changqing District, Jinan, Shandong 250355, China, Tel +86-531-89628077, Email: make19880710@163.com.

(C) The author(s). This is an open access article distributed under the terms of the Creative Commons Attribution License (https://creativecommons.org/licenses/by/4.0/). See http://ivyspring.com/terms for full terms and conditions.

Received: 2020.11.11; Accepted: 2021.03.30; Published: 2021.04.16

\begin{abstract}
Endometriosis (EMs) is recorded as Zheng Jia in traditional Chinese medicine (TCM) books. Guizhi Fuling Pills (GFPs), a classic prescription for promoting blood circulation and removing blood stasis, is widely used for women's blood stasis diseases represented by Zheng Jia. At present, it has been applied to treat EMs in clinical settings. In this review, we systematically summarized the active ingredients and pharmacological mechanism of five Chinese herbs contained in GFPs and clinical applications of GFPs. The potential pathways of GFPs in the treatment of EMs were explored through network pharmacology. The current researches results indicate that the mechanisms of GFPs in the treatment of EMs mainly include acesodyne, anti-inflammation and improvement of hemodynamics. The main active compounds that are responsible for pharmacological effects in five Chinese herbs are paeonol, pachymic acid, cinnamaldehyde, amygdaloside and Paeoniflorin. This review can lay the foundation and identify the research direction for the development of GFPs as a new drug therapy for the treatment of EMs.
\end{abstract}

Key words: Endometriosis; Zheng Jia; Guizhi Fuling Pills; traditional Chinese medicine; pharmacological

\section{Introduction}

Endometriosis (EMs) is defined as the presence and growth of endometrial-like tissue outside the uterus, which is mainly characterized by dysmenorrhea, dyspareunia, pelvic pain and infertility $[1,2]$. It is estimated that the prevalence of EMs is $6-10 \%$ and the majority of suffer are women aged 25-35 years old [1, 3]. Unfortunately, the understanding of its pathogenesis is insufficient and explicit. It is possibly connected with retrograde menstruation phenomenon, estrogen dependence, progesterone resistance and inflammation [4, 5]. Hormonal therapy and surgical therapy are currently common therapy for EMs. However, hormone compounds could only temporarily inhibit the secretion of endogenous ovarian hormones $[6,7]$. In addition, long-term use of hormones may lead to impaired ovarian reserve function, interference with ovulation, abnormal uterine bleeding and infertility $[6,7]$. A five-year follow-up study showed that without drug-assisted treatment, the recurrence rate of patients treated by surgery was as high as $40-50 \%$ [8]. Therefore, it is particularly important to seek new drugs that can treat endometriosis or relieve pain without interfering with ovulation.

TCM pills are one of the main forms of traditional Chinese medicine preparations, which are mostly applied in the clinical treatment of chronic diseases and regulation of body function [9]. For example, Liuwei Dihuang Pills was used for curing kidney deficiency as well as Lizhong Pills with the functions of warming and strengthening spleen [9]. The gynecology of TCM has a history of 3,000 years. In the book Synopsis of the Golden Chamber written by Zhang Zhongjing in the Eastern Han Dynasty, the prevention and treatment of women's pregnancy diseases, postpartum diseases and other common diseases had been systematically recorded. Moreover, the book summarized a wealth of treatment 
experience and medication principles on gynecology diseases. Classic famous prescription GFPs is a representative TCM pill in this book. Its efficacy is to promote blood circulation, remove blood stasis, eliminate abdominal mass and stop vaginal bleeding. Recently, it has been mainly used in the clinical treatment of EMs as well as adenomyosis (AM) [10, 11]. In order to understand the pharmacological mechanism and clinical application of GFPs in the treatment of EMs, we reviewed and summarized the previous clinical and animal studies concerning the treatment of EMs with GFPs, with the aim of clarifying the advantages of TCM in the treatment of gynecological diseases.

\section{Endometriosis and Zheng jia}

There is no relevant record about EMs in TCM books. According to its clinical manifestations, it can be considered as Zheng Jia in TCM, which was first recorded in Huangdi Neijing. Chinese medicine scholars named all the lumps in the human body as Zheng Jia [12]. Synopsis of the Golden Chamber had discussed in detail that lower abdominal pain, abdominal mass and irregular vaginal bleeding were the main clinical manifestations of Zheng Jia. Thus, Zheng Jia, as a gynecological disease, usually refers to the agglomeration in female lower abdomen accompanied by abdominal distension, pain, vaginal bleeding and other symptoms. AM, EMs, polycystic ovarian syndrome (PCOS) and reproductive system tumors all can be regarded as Zheng Jia according to its symptoms and pathological signs [13]. Further, the pathogenesis of Zheng Jia is also similar to the EMs in TCM. TCM theory explains that the pathogenesis of Zheng Jia is mainly related to blood accumulation and qi stagnation in the uterus, which form lumps over time. TCM proposes that EMs is caused by retrograde menstruation, which results in blood stasis blocking the uterus for a long time. Thus, blood stasis is the core pathogenesis and pathological essence of both Zheng Jia and EMs.

Based on the pathogenesis of Zheng Jia, the relevant therapeutic methods are suggested in the Synopsis of the Golden Chamber "Chapter 20, the pluse, syndrome and treatment of women during pregnancy". Promoting blood circulation, removing blood stasis, warming and dredging meridians are the core therapeutic methods. Subsequently, the classic prescription GFPs is established. The prescription is composed of 5 traditional Chinese herbs: Cassia Twig (Cinnamomum cassia Presl), Poria Cocos (Poria $\operatorname{cocos}($ Schw.)Wolf), Peach Kernel (Prunus persica (L.) Batsch), Red Peony Root (Paeonia veitchii Lynch) and Cortex Moutan (Paeonia suffruticosa Andr.). Poria Cocos plays a role in infiltrating dampness and strengthening spleen. Peach Kernel and Red Paeony root have effects on promoting blood circulation and relieveing pain. Cortex Moutan is commonly used to clearing the heat caused by blood stasis [14]. The combination of the five herbs can maximize the functions in the treatment of EMs [15].

\section{Pharmacological study on active components of five Chinese herbs of GFPs}

\section{Cassia Twig (CT)}

CT which is the dried twig of Cinnamon was first recorded in the Shen Nong's herbal Classic and was commonly used in clinic. CT has the efficacy of sweating and slackening muscles, warming and dredging meridians, and assisting yang and transforming qi [16]. CT contains a lot of different ingredients, such as benzaldehyde, trans-cinnamaldehyde, organic acids, tannins, sugars, steroids and other components. While the most important ingredient in the volatile oil of $\mathrm{CT}$ is cinnamaldehyde [17, 18]. Pharmacological research demonstrated that the volatile oil of CT played a positive role in diminishing inflammation, relieving pain, promoting blood circulation and resisting platelet aggregation $[17,19]$. The occurrence of EMs was associated with the increase of cyclooxygenase-2 (COX-2), vascular endothelial growth factor (VEGF) and tumor necrosis factor alpha (TNF-a) contents as well as changes in NF-kB signal pathway. Chao el. indicated that cinnamaldehyde could reduce inflammatory reaction by activating NF-kB signal pathway and reducing the production of interleukin-1 beta (IL-1 $\beta$ ) and TNF-a [20]. Yao el. confirmed that volatile oil of CT could reduce the expression of VEGF, COX-2 and other factors in ovarian tissue of rats, which could effectively treat infertility [21]. Furthermore, in the clinical treatment of EMs, CT is often used in combination with other traditional Chinese herbs instead of single application. Studies proved that the compatibility of $\mathrm{CT}$ with poria cocos could increase the dissolution rate of cinnamaldehyde by nearly 3 times, which could enhance the efficacy of $\mathrm{CT}$ in promoting blood circulation and dredging collaterals $[22,23]$. Cooperating with peach kernel, the anticoagulant effect is stronger than that of $\mathrm{CT}$ alone [24]. In conclusion, CT can be applied to treat EMs based on its anti-inflammatory and anticoagulant effects.

\section{Poria Cocos (PC)}

PC is the dry sclerotium of Polyporaceae [25]. In TCM clinic, PC which has the effects of eliminating dampness, invigorating spleen, calming heart and 
tranquilizing mind is applied in many settings [26]. Pharmacological researches demonstrated that PC had an active impact on againsting cancer, regulating immune function, diminishing inflammation and preventing oxidization. Furthermore, report found that triterpenes and polysaccharides were the main active components in PK that exerted pharmacological effects [27]. Among polysaccharides, pachyman is one of the most important active ingredients, which can regulate immune function, resist inflammation and inhibit tumor. The mechanism of pachyman regulating immune function might be depend on enhancing Natural killer (NK) cell activity and regulating the levels of inflammatory cytokines such as interleukin-2 (IL-2) and TNF-a [28]. Besides, triterpenes of PC also plays a crucial role in anti- inflammatory effect. There were evidences to support that it could inhibit the expression of IL-1 $\beta$ through MAPK, PI3K/Akt and NF-kB signaling pathways [29]. Research indicated that it was also related to bidirectional regulation of MAPK signaling pathways to inhibit macrophage inflammatory factor expression [30]. Moreover, it could induce the release of prostaglandin I2 (PGI2) by upregulation of COX-2 in the MAPK signaling pathway to maintain vascular homeostasis [31]. Additionally, pachymic acid could reduce the excessive secretion of nitric oxide, endothelin -1 (ET-1) and thromboxane A2 to inhibit platelet aggregation [32]. Meanwhile, EMs involved with multiple signal transduction pathways, such as MAPK, NF-kB, and was closely associated with inflammatory response [33]. Lee el. proposed that
A


D

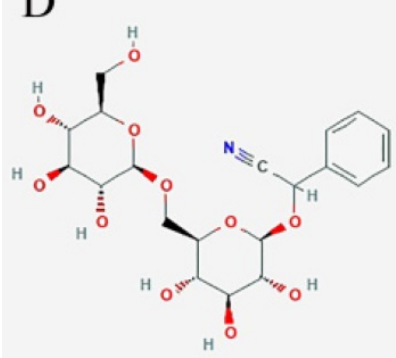

$\mathrm{E}$

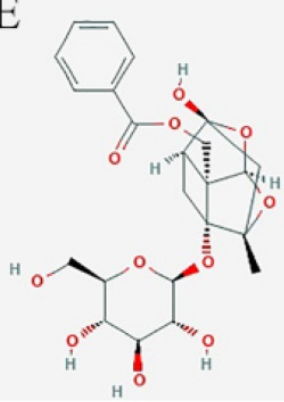

Figure 1. Structural formula of five important compounds in five Chinese herbs of GFPs. A: paeonol; B: pachymic acid; C:cinnamaldehyde; D: amygdaloside; E: Paeoniflorin.
p38MAPK which was phosphorylated by IL-1 was expressed in both ectopic and eutopic endometrium and stimulated inflammatory cells to secrete more interleukin-6 (IL-6), interleukin-8 (IL-8), COX-2 and other inflammatory mediators in EMs patients [34]. Khan el. suggested that lipopolysaccharide (LPS) stimulated the production of high levels of TNF-a, IL-6 and IL-8 through activating the NF-kB pathway in the ectopenic endometrium stromal cells [35]. Therefore, PC can be applied to treat EMs based on its anti-inflammatory and immune function regulating effects.

\section{Peach Kernel (PK)}

PK is the dried mature seed of Rosaceae plant peach or mountain peach, which was first recorded in Shen Nong's Herbal Classic. The main efficacy of PC includes promoting blood circulation, removing blood stasis, relaxing bowels as well as relieving cough and asthma [36]. In TCM clinical setting, it is often used for the treatment of amenorrhea, dysmenorrhea, abdominal mass, traumatic injury, intestinal dryness, constipation, cough, asthma and other diseases [37]. Pharmacological studies demonstrated that PK mainly contained fat-soluble substances, proteins, sterols, glycosides, flavonoids, phenolic acids and other active ingredients, which had the effects of anticoagulant, antithrombotic, anti-inflammatory, preventing liver fibrosis and enhancing immunity [38]. Report from Zhu el confirmed that water extract derived from PK could effectively inhibit platelet aggregation and it was much more effective than the amygdalin and the fat oil of PK [39]. Glyceryl

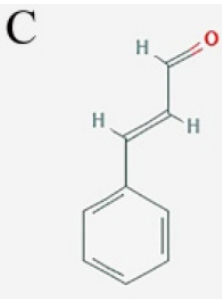
trioleate contained in the oil of PK had anticoagulant activity. And the coagulation time extension rate of it was $37 \%$ [40]. Amygdalin, the main chemical component in PK, exerted anti-inflammatory activity by inhibiting the expression of COX-2 and inducible nitric oxide synthase in mouse BV2 microglia induced by LPS [41]. Further, it could effectively relieve inflammatory pain, and the mechanism of action was connected with c-fos and inflammatory factors [42]. Thus, PK is beneficial to the treatment of EMs, owning to the functions of pain and inflammation relieving. 


\section{Red Peony Root (RPR)}

The medicinal part of Paeonia lactiflora is rhizome, which is divided into RPR and Radix Paeoniae Alba (RPA). TCM theory consider that RPA is beneficial to nourish yin and tonify deficiency, while RPR is suitable for promoting blood circulation, removing blood stasis and relieving pain [43]. Because Zheng Jia is blood stasis syndrome, the Paeonia lactiflora in GFPs should refer to RPR. Pharmacological researches indicated that the main components of RPA and RPR were monoterpene, glycoside, tannin and organic acids [44]. Although the chemical compositions of RPA and RPR are similar, they are not completely the same. The content of paeony glycoside in RPR was much higher than that of RPA, however, RPA contained more paeony lactone glycoside [44, 45]. Report supported that paeony glycoside had significant analgesic effect, and its analgesic effect was better than that of paeony lactone glycoside [46]. There was evidence showed that excessive prostaglandin released from ectopic endometrium tissue cause myometrial hypertonus and secondary ischemia, resulting in dysmenorrhea in patients [47, 48]. Meanwhile, research demonstrated that paeony glycoside effectively reduced the twisting times of mouse pain model caused by acetic acid induced writhing, and the analgesic mechanism might be related to increasing the level of $\beta$-endorphin in cerebral cortex and serum and reducing the secretion of prostaglandin estradiol (E2) [46]. Apart from this, study proved that paeony glycoside could improve the blood stasis state of rats with acute blood stasis syndrome by reducing the whole blood viscosity and regulating the balance of ET-1 and NO, and its effect was stronger than that of paeony lactone glycoside [49]. Thus, RPR can be applied to treat EMs based on its analgesic and blood circulation promoting effects.

\section{Cortex Moutan (CM)}

CM was first published in Shen Nong's Herbal Classic. Its medical effectiveness is clearing heat and cooling blood, promoting blood circulation and removing blood stasis [50]. CM contained complex chemical components. The paeonol, paeony glycoside and benzoyl paeoniflorin were the main active compounds [51]. Paeony glycoside could alleviate pain obviously [46]. Besides, paeonol significantly inhibited the proliferation and differentiation of tumor cells. Study confirmed that the anti-tumor mechanism of paeonol was based on its inhibition of expression of cyclooxygenase -2 (COX-2) gene [52]. Correspondingly, inflammation is a typical feature of EMs, and the contents of COX-2, NF-K B and other cytokines significantly increased in ectopic endometrium [53-55]. Consequently, the anti-inflammatory effect of CM is suitable for EMs. In addition, research proposed that paeonol reduced inflammatory response by decreasing the content of IL-1 and IL-6 in the serum of EMs rat model and improve blood viscosity [56].Moreover, evidence supported that benzoyl paeoniflorin could play an anti-inflammatory role by inhibiting COX-1 and COX-2 activities [57].

\section{Clinical application of GFPs in the treatment of EMs}

In the clinical treatment of EMs, GFPs is often changed into decoction, and the proportions of $\mathrm{CT}$, PC, PK, RPR, CM is equal and the usual dose of five Chinese herbs in the prescription is $12 \mathrm{~g}$ [58]. The Chinese herbs were decocted into $500 \mathrm{ml}$ liquid medicine, and take it in the morning and evening. Besides, for convenience of taking medicine, the herbs were also encapsulated for administration at $0.31 \mathrm{~g}$ per capsule. The method of administration is to take three capsules three times a day [59].

In recent years, a series of studies proved that treating EMs with GFPs alone or in combination with western medicine had achieved satisfactory curative effect and was considered as an alternative drug for treating EMs [60-62]. GFPs, taken three pills at a time, three times a day, combined with mordern medicine could significantly reduce the levels of Leptin, VEGF and IL-8 in serum of patients with EMs and improve ovarian function. In addition, it has obvious effect on inhibiting the growth of cystic mass and relieving dysmenorrhea [63]. Zhang el. [64, 65] conducted a randomized controlled trial to compare the clinical efficacy of mifepristone and GFPs combined with mifepristone in the treatment of EMs. The test data indicated that GFPs combined with mifepristone had a better reduction in the levels of CA125, CA199, VEGF, superoxide dismutase (SOD), IL-6, IL-8, TNF- $a$, hs-CRP than mifepristone alone. The clinical effective rate of the test group was $94.33 \%$, significantly higher than that of the control group $75.47 \%$. Moreover, GFPs has long-lasting effect and low recurrence rate in the treatment of EMs. A 3-month continuous treatment experiment compared the GFPs with the Danazol. The result showed the recurrence rate of GFPs group was $17.4 \%$ one year after drug withdrawal, which was lower than $31.8 \%$ of the Danazol group [66]. Furthermore, GFPs combined with other comprehensive treatment of TCM also applied to treat EMs, for example, TCM enema and acupuncture. Study proposed that the total effective rate of the combination of GFPs and TCM enema for EMs was 96\%, which was higher than $80 \%$ of the control group [67]. In addition, 
acupuncture aided GFPs can not only get a significant therapeutic effect on EMs but also has fewer side effects. Compared with the control group, the total effective rate is $79.49 \%$ and the effective rate for pain relief was $76.92 \%$, which was higher than that of the control group $(\mathrm{P}<0.05)$ [68]. As a result, under the guidance of syndrome differentiation and treatment theory of TCM, GFPs are appropriated for EMs of blood stasis type $[69,70]$.

$\mathrm{AM}$ is a common gynecologic disease, which is caused by invasion of endometrial glands and stroma into myometrium to form diffuse or localized lesions $[71,72]$. There are many similarities between AM and EMs in clinical manifestations, pathogenesis and treatment methods. Research demonstrated that GFPs also has good therapeutic effects on AM [73]. Relevant studies confirmed that GFPs combined with western medicine was more effective than oral western medicine in the treatment of AM. A randomized clinical trial was designed to compare the clinical effects of GFPs and gestrinone. Patients in control group were given gestrinone orally, $2.5 \mathrm{mg} /$ time, three times a week. Observation group were treated with GFPs on the basis of the control group. The specific dosage and usage of the drug are as follows: CT, CM 12 g each, PK 10 g, RPR 8 g, and PC 9 g, taken orally twice in the morning and evening. The specific dosage and usage of the drug are as follows: CT, CM $12 \mathrm{~g}$ each, PK $10 \mathrm{~g}$, RPR 8 g, and PC 9 g, taken orally twice in the morning and evening. The results indicated that the clinical effective rate of the experimental group was $91.7 \%$ higher than that of the control group 70.8\%, and the levels of IL-6, PGE-2 and ET were lower than those of the control group [74]. In addition, the clinical efficacy of radiofrequency ablation plus GFPs was significantly effective than that of radiofrequency ablation alone, and had the advantages of reducing recurrence rate and ameliorating clinical symptoms [75].

\section{Prediction of potential mechanism of GFPs in treating EMs}

TCM has the characteristics of multi-component, multi-target and multi-pathway, as a result, it is difficult to analyze the complex mechanism of TCM only by traditional experimental methods [76]. Network pharmacology, as a powerful method integrating system biology, bioinformatics and multiple pharmacology is a reliable approach to explore the potential therapeutic mechanism of herbs [77]. It clarifies the complex interactions among genes, proteins and compounds related to diseases and drugs at the network level.

Based on the principle of network pharmacology, we explored the mechanism of GFPs therapy for EMs. Firstly, we confirmed 102 targets of GFPSs from websites TCMSP (http://tcmspw.com/ tcmsp.php) and SymMap (http://www.symmap. org/). Second, 304 endometriosis-related genes were obtained from databases DisGeNET (http://www. disgenet.org/) and GeneCards (https://www. genecards.org/). Subsequently, 26 intersection genes were obtained by Venn diagram. Kyoto Encyclopedia of Genes and Genomes (KEGG) analysis was explored to speculate the potential pathway of GFPs treatment for EM. Table 1 presents the 9 concerned pathways. The results of network pharmacology research supported that the potential mechanism of GFPs in the treatment of EMs might be inflammatory pathways, such as, TNF signaling pathway $(\mathrm{p}<0.01)$ and NF-kappa B signaling pathway $(\mathrm{p}<0.01)$.

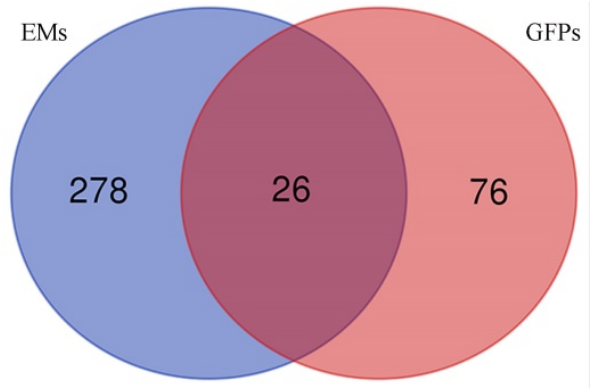

Figure 2. The intersection genes of GFPs and Ems.

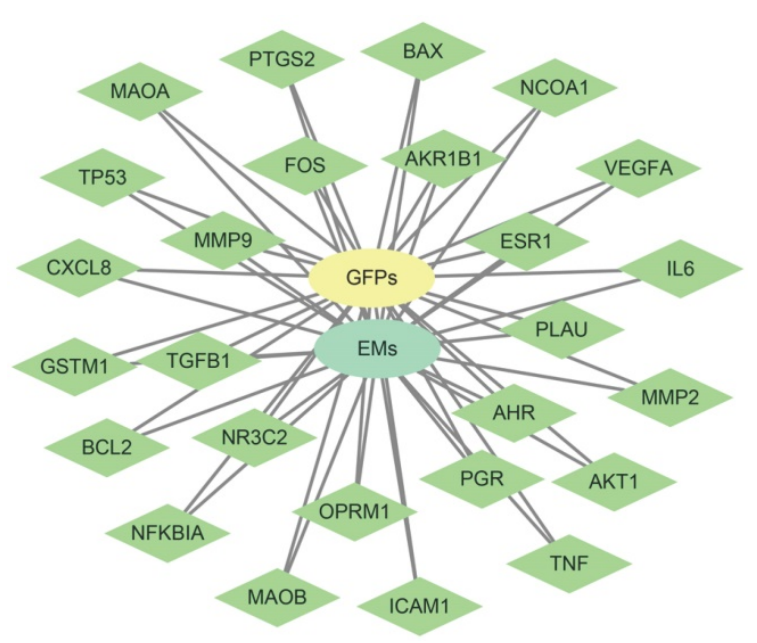

Figure 3. 26 disease targets have established networks with GFPs and EMs. Green node represents intersection gene, yellow node represents GFPs, and blue nod represents EMs.

Table 1. The information of 11 potential target pathways

\begin{tabular}{llll}
\hline ID & Term & Count & P-value \\
\hline hsa05200 & Pathways in cancer & 13 & $1.48 \mathrm{E}-09$ \\
hsa04668 & TNF signaling pathway & 8 & $5.03 \mathrm{E}-08$ \\
hsa05205 & Proteoglycans in cancer & 9 & $2.19 \mathrm{E}-07$ \\
hsa04064 & NF-kappa B signaling pathway & 7 & $3.86 \mathrm{E}-07$ \\
hsa04210 & Apoptosis & 6 & $1.88 \mathrm{E}-06$ \\
hsa04915 & Estrogen signaling pathway & 6 & $1.91 \mathrm{E}-05$ \\
hsa05202 & Transcriptional misregulation in cancer & 5 & 0.002435561 \\
hsa05206 & MicroRNAs in cancer & 6 & 0.002657271 \\
hsa04066 & HIF-1 signaling pathway & 4 & 0.004310234 \\
\hline
\end{tabular}


Table 2. The TCM efficacy and main components of five Chinese herbs in GFPs

\begin{tabular}{lll}
\hline $\begin{array}{l}\text { Chinese } \\
\text { herbs }\end{array}$ & TCM efficacy & Main components \\
\hline $\begin{array}{l}\text { Cassia } \\
\text { Twig }\end{array}$ & $\begin{array}{l}\text { Sweating and slackening muscles, } \\
\text { warming and dredging meridians, and } \\
\text { assisting yang, transforming qi }\end{array}$ & $\begin{array}{l}\text { Benzaldehyde, } \\
\text { trans-cinnamaldehyde, } \\
\text { organic acids, tannins, sugars, } \\
\text { steroids }\end{array}$ \\
$\begin{array}{lll}\text { Poria } \\
\text { Cocos }\end{array}$ & $\begin{array}{l}\text { Eliminating dampness, invigorating } \\
\text { spleen, calming heart, tranquilizing mind } \\
\text { promoting blood circulation, removing }\end{array}$ & $\begin{array}{l}\text { Triterpenes, polysaccharides } \\
\text { fat-soluble substances, }\end{array}$ \\
Kernel & $\begin{array}{l}\text { blood stasis, relaxing bowels, relieving } \\
\text { cough and asthma }\end{array}$ & $\begin{array}{l}\text { proteins, sterols, glycosides, } \\
\text { flavonoids, phenolic acids }\end{array}$ \\
Red & $\begin{array}{l}\text { Promoting blood circulation, removing } \\
\text { Peony }\end{array}$ & $\begin{array}{l}\text { Monoterpene, glycoside, } \\
\text { bannin,organic acids }\end{array}$ \\
$\begin{array}{l}\text { Root } \\
\text { Cortex }\end{array}$ & $\begin{array}{l}\text { Clearing heat, cooling blood, promoting } \\
\text { Moutan }\end{array}$ & $\begin{array}{l}\text { blood circulation, removing blood stasis } \\
\text { and benzoyl paeoniflorin }\end{array}$ \\
\hline
\end{tabular}

\section{Conclusion and Prospect}

Treatment of EMs is a challenge in the field of gynecology. The accompanying dysmenorrhea and infertility have troubled women of childbearing age. However, the current treatment approaches have the defects of side-effect and high recurrence rate. Therefore, it is essential to develop drugs that can target the pathogenesis of EMs and effectively relieves the symptoms of patients without affecting ovulation and pregnancy functions. TCM is a precious resource, and many Chinese herbal medicines are worth exploring. Many clinical practices have proved the advantages of TCM in the treatment of disease, and have gradually attracted worldwide attention. EMs syndrome differentiation belongs to the category of blood stasis syndrome in TCM. GFPs has been used for the treatment of EMs in recent years as a famous classical Chinese medicine prescription commonly applied for the treatment of women's blood stasis disease in history. In order to deeply understand the effective components, mechanism and advantages of GFPs in clinical treatment of EMs, the articles concerned with pharmacology, animal experiments and clinical trials are classified and sorted out. At present, the main functions of medicine monomer in GFPs were focused on anti-tumor, anti-inflammatory, analgesic and hemodynamic improvement. The network pharmacology research also indicated that the potential mechanism of GFPs in the treatment of EMs might be inflammatory pathways, such as, TNF signaling pathway and NF-kappa B signaling pathway. However, it did not fully clarify the mechanism of GFPs as EMs therapeutic drug. For example, the understanding of medicine composition is insufficient. The related researches on medicine ingredients are few and lack pertinence to EMs. The clinical control experiment cases are few. Currently, more and more attention is being paid to the multidisciplinary integration research of TCM. For example, on the basis of classic famous prescriptions of TCM and combined with modern pharmacological research and evidence-based medicine, innovative addition and subtraction of Chinese herbs were carried out. The clinical efficacy of Sanjie Zhentong Capsule which is established by clinical experiences had been proved to be effective in the treatment of EMs [78]. Studies proved that the active ingredients Verticinone and Verticine in Sanjie Zhentong Capsule had significant analgesic and anti-inflammatory activities [79]. Besides, Apigenin had estrogen-like effect on animals, and it inhibited ovarian secretion of estrogen and progesterone [80]. Thus, multidisciplinary integration will all help promote the modernization of TCM and the development of gynecology of TCM.

\section{Abbreviations}

EMs: endometriosis; TCM: traditional Chinese medicine; GFPs: Guizhi Fuling pills; AM: adenomyosis; PCOS: polycystic ovarian syndrome; CT: Cassia Twig; PC: Poria Cocos; PK: Peach Kernel; RPR: Red Peony Root; RPA: Radix Paeoniae Alba; CM: Cortex Moutan; LPS: lipopolysaccharide; COX-2: cyclooxygenase-2; VEGF: vascular endothelial growth factor; TNF-a: tumor necrosis factor alpha; IL-1 $\beta$ : interleukin-1 beta; NK: natural killer; IL-2: interleukin-2; PGI2: prostaglandin I2; IL-6: interleukin-6; IL-8: interleukin-8; ET-1: endothelin-1; E2: estradiol; SOD: superoxide dismutase; KEGG: Kyoto Encyclopedia of Genes and Genomes.

\section{Acknowledgements}

We are grateful to the technical team of Shandong Province Universities' Development Plan for Youth Innovation Teams.

\section{Funding}

This work was supported by the Shandong Co-Innovation Center of Classic TCM Formula, Shandong Province Universities' Development Plan for Youth Innovation Teams (2019-9-202). This project was also supported by National Nature Science Foundation of China (81903948, 81674014, 81873330), Shandong Provincial Natural Science Foundation (ZR2019BH027), Shandong Province University Scientific Research Project (J18KZ014), Taishan Scholar Project of Shandong Province (tsqn201909185).

\section{Authors' contributions}

XW, YXS, ZLW, YFW and LX contributed to literature review and data analyses. $\mathrm{KM}$ and WS contributed to the project design and paper writing. All authors have read and approved the final version of the manuscript. 


\section{Availability of data and materials}

All the data used to support the findings of this study are available from the corresponding author upon reasonable request.

\section{Competing Interests}

\section{interest exists.}

\section{References}

1. Vercellini P, Vigano P, Somigliana E, Fedele L. Endometriosis: pathogenesis and treatment. Nature reviews Endocrinology. 2014; 10: 261-75.

2. Johnson NP, Hummelshoj L, Adamson GD, Keckstein J, Taylor HS, Abrao MS, et al. World Endometriosis Society consensus on the classification of endometriosis. Human reproduction (Oxford, England). 2017; 32: 315-24.

3. Ferrero S, Arena E, Morando A, Remorgida V. Prevalence of newly diagnosed endometriosis in women attending the general practitioner. International journal of gynaecology and obstetrics: the official organ of the International Federation of Gynaecology and Obstetrics. 2010; 110: 203-7.

4. Barra F, Scala C, Ferrero S. Current understanding on pharmacokinetics, clinical efficacy and safety of progestins for treating pain associated to endometriosis. Expert opinion on drug metabolism \& toxicology. 2018; 14: 399-415.

5. Burney RO, Giudice LC. Pathogenesis and pathophysiology of endometriosis. Fertility and sterility. 2012; 98: 511-9.

6. Barra F, Grandi G, Tantari M, Scala C, Facchinetti F, Ferrero S. A comprehensive review of hormonal and biological therapies for endometriosis: latest developments. Expert opinion on biological therapy. 2019; 19: 343-60.

7. Gheorghisan-Galateanu A. Hormonal Therapy in Women of Reproductive Age with Endometriosis: an Update. Acta Endocrinologica (Bucharest). 2019; 15: $276-81$

8. Guo SW. Recurrence of endometriosis and its control. Human reproduction update. 2009; 15: 441-61.

9. Yu HY, Cai CL, Du QW, Zhang CC, Fu JL, Sun DD, et al. Research progress of traditional Chinese medicine pills. Journal of Pharmaceutical Research. 2019; 38: $424-6+30$.

10. Ding N, Wang X, Xia XJ. Effects of Guizhi Fuling Pill on Serum IL-2, IL-8, VEGF and Ovarian Function in Patients with Endometriosis. Journal of Liaoning University of Traditional Chinese Medicine. 2019; 21: 110-3.

11. Guo JL. Effects of mifepristone combined with Guizhi Fuling Capsules on treating adenomyosis in 34 cases. Chongqing Medicine. 2011; 40: 673-5.

12. Gu SC, Yang BC. An analysis of the scope of symptoms and treatment of an accumulation of symptoms. JiangSu Journal of Traditional Chinese Medicine. 2017; 49: 11-3

13. Wu CX, Liu WE, Hou MH. A brief analysis of zhang xichun's experience in treating women's syndrome and its modern application. Clinical Journal of Traditional Chinese Medicine. 2019; 31: 2068-70.

14. Zhu L, Li D, Ni J, Zhang W, Han H. An analysis of prescriptions for the treatment of women's syndrome in synopsis of golden chamber. Chinese Journal of Information on Traditional Chinese Medicine. 2016; 23: 100-1.

15. $\mathrm{Hu} \mathrm{NN}$, Zhao $\mathrm{C}$, Zhang JQ, Wang $\mathrm{X}$, Yang DX, Wu XH. Clinical and mechanism research progress of cassia twigi tuckaing pill in the treatment of endometriosis. Acta Chinese Medicine and Pharmacology. 2017; 45: 104-7.

16. Wang $\mathrm{YH}, \mathrm{Yu} \mathrm{CN}$. Textual research on the efficacy of cassia twig. Asia-Pacific Traditional Medicine. 2018; 14: 66-7.

17. Xu Y, Su SL, Wang TJ, Duan JA, Wang ZZ. Research progress on chemical constituents and pharmacological activities of cassia twig. Journal of Chinese Medicinal Materials. 2013; 36: 674-8.

18. Shen $\mathrm{Q}$, Chen FL, Luo JB. Analysis of chemical constituents of volatile oil from cassia twig and cinnamon by gc-ms. Science of Chinese Materia Medica. 2002: 257-8.

19. Xu F, Wang DJ, Wang F, Wen TQ, Sang WT, Zeng N. Progress in the pharmacological action of volatile oil from cassia twig. China Journal of Traditional Chinese Medicine and Pharmacy. 2016; 31: 4653-7.

20. Chao LK, Hua KF, Hu HY. Cinnamaldehyde inhibits proinflammatory cytokines secretion from monocytes/macrophages through suppression of intracellular signaling. Food and Chemical Toxicology. 2008; 46: 220-31.

21. Yao F, Cao JX, Wang RG. Effects of volatile oil from cassia twig on expression of VEGF, cox-2 and MVD in ovary of PCOS rats. Guiding Journal of Traditional Chinese Medicine and Pharmacy. 2012; 18: 15-7.

22. Cao WX, Wang XQ, Yu XM, Wei T, Li YX, Xiang Z. The application of interactive moving window factor method in the study of volatile oil composition and dissolution rate before and after the combination of cassia twig and tuckahoe. Chinese Journal of Chinese Meteria Medicine. 2008: 2056-60.

23. Yu CL, Du ZC, Hao EW, Wei W, Guo ZW, Hou XT, et al. Research progress on chemical constituents and pharmacological effects of four kinds of osmanthus drugs with different effects. Chinese Journal of Experimental Traditional Medical Formulae. 2020; 26: 226-34.

24. Zhou B. Study on the effect of osmanthus peach kernel and its compatibility on anticoagulation. Yunnan Journal of Traditional Chinese Medicine and Materia Medica. 2014; 35: 62-3.

25. Zhang L, Liu Q. Research progress on the preparation technology and pharmacological action of tuckahoe polysaccharide. Chinese Journal of Experimental Traditional Medical Formulae. 2006; 12: 61-4.

26. Feng YL, Zhao YY, Ding F, Diao ZH, Tian T, Zhou F, et al. Chemical constituents and pharmacological research progress of poria cocos skin (I). China Journal of Chinese Materia Medica. April,2013; 38: 1098-102.

27. Zhang N, Li ZX, Li J, Liu J, Dai JM, Li YW, et al. Advances in the study of chemical constituents and biological activities of tuckahoe. World Science and Technology/Modernization of Traditional Chinese Medicine and Materia Medica. 2019; 21: 220-33.

28. Zhang M, Chiu L, Cheung P, Ooi V. Growth-inhibitory effects of a $\beta$-glucan from the mycelium of Poria cocoson human breast carcinoma MCF-7 cells: Cell-cycle arrest and apoptosis induction. Oncology Reports. 2006; 15: 637-43.

29. Wu QQ, Wang J, Cui S, Chen SS, Lei Y, Li SH, et al. Oleanolic acid inhibits lipopolysaccharide induced inflammatory response in RAW264.7 cells. Journal of Yangzhou University (Agriculture and Life Sciences Edition). 2017; 38: 27-31.

30. Lian JJ, Cheng BF, Gao YX, Zhang YP, Ma SP, Zhang BB, et al. The inhibitory effect of oleanolic acid on il-1-induced inflammatory response of SW982 cells. Acta pharmaceutica sinica. 2016; 51: 1711-6.

31. J M-G, R R-R, M G-D, C R, MD H, V R-G, et al. Oleanolic acid induces prostacyclin release in human vascular smooth muscle cells through a cyclooxygenase-2-dependent mechanism. The Journal of nutrition. 2008; 138: 443-8

32. Hong Chao Z, Li Yun G, Guang Li F, Zhong Jie L, Xiang M. Effects of pachymic acid on the secretion of cytokines from rat intestinal microvascular endothelial cells induced by SLT-IIe. Chinese Veterinary Science. 2008; 39: 884-8

33. Zhuang MF, Yang Y, Cao Y, Yuan XF, Zhu Y, Xie SW. The research progress of signal pathways related to the pathological mechanism of endometriosis. Chinese Journal of Reproduction and Contraception. 2016; 36: 214-22+44.

34. Lee D, Kim S, Joo J, Kim H, Na Y, Kwak J, et al. Effects of $17 \beta$-estradiol on the release of monocyte chemotactic protein-1 and MAPK activity in monocytes stimulated with peritoneal fluid from endometriosis patients. The journal of obstetrics and gynaecology research. 2012; 38: 516-25.

35. Khan KN, Kitajima M, Inoue T, Fujishita A, Nakashima M, Masuzaki $H$. 17beta-estradiol and lipopolysaccharide additively promote pelvic inflammation and growth of endometriosis. Reprod Sci. 2015; 22: 585-94.

36. $\mathrm{Xu} \mathrm{XH,} \mathrm{Li} \mathrm{T,} \mathrm{Wang} \mathrm{YT,} \mathrm{Lu} \mathrm{JJ.} \mathrm{Research} \mathrm{progress} \mathrm{of} \mathrm{peach} \mathrm{kernel.} \mathrm{Chinese}$ Traditional and Herbal Drugs. 2015; 46: 2649-55.

37. Zhao YJ, Niu K, Tang DZ, Liang QQ, Shu B, Li CG, et al. Recent research on pharmacological action of peach kernel. Liaoning Journal of Traditional Chinese Medicine. 2015; 42: 888-90.

38. Wang RF, Fan LG, Gao WY, Zhang JY. Research progress on chemical constituents and pharmacological activities of peach kernel. Drugs \& Clinic. 2010; 25: 426-9.

39. Zhu XX, Zhu F, Shi RS, Zhen C. Study on the platelet aggregation effect of peach seed and antihexis extracts in rats. Research of Traditional Chinese Medicine. 2000: 44-5.

40. Wilson R SJ. Chain separation of monounsaturated fatty acid methyl esters by argentation thin-layer chromatography. Journal of chromatography A. 2001; 905: 251-7

41. Yang HY, Chang HK, Lee JW, Kim YS, Kim H, Lee MH, et al. Amygdalin suppresses lipopolysaccharide-induced expressions of cyclooxygenase-2 and inducible nitric oxide synthase in mouse BV2 microglial cells. Neurological research. 2007: S59-64.

42. Hwang HJ, Kim P, Kim CJ, Lee HJ, Shim I, Yin CS, et al. Antinociceptive effect of amygdalin isolated from Prunus armeniaca on formalin-induced pain in rats. Biological \& pharmaceutical bulletin. 2008; 31: 1559-64.

43. Zhang YL, Chen X, Gao QL, Zheng Jj. An analysis of Shaoyao in Shanghan Zabing Lun. Clinical Journal of Chinese Medicine. 2019; 11: 17-9.

44. Zhang JJ, Li W, Wang LL, Huang YF, Wang C, Wang JX, et al. Varieties, functions and clinical applications of Chishao and Baishao:a literature review. China Journal of Chinese Materia Medica. 2013; 38: 3595-601.

45. Zhou HL, Xu SJ, Zhou RL, Ma L, Zhan JJ, Yang L. Analysis of chemical constituents of Radix Paeoniae Alba and Radix Paeoniae Rubra by high performance liquid chromatography-time of flight tandem mass spectrometry. Journal of Chinese Medicinal Materials. 2018; 41: 1637-40.

46. Wu L, Wang LL, Fei WT, Wang S, Hou Y, Zhan JJ. Analgesic effect of paeoniflorin and albiflorin on acetic acid induced writhing mice and the expression of $\beta$-EP and PGE China Journal of Traditional Chinese Medicine and Pharmacy. 2018; 33: 915-8.

47. Howard FM. Endometriosis and mechanisms of pelvic pain. Journal of minimally invasive gynecology. 2009; 16: 540-50

48. Vercellini P. Endometriosis: what a pain it is. Seminars in reproductive endocrinology. 1997; 15: 251-61.

49. Zhang L, Zhao BJ, Yuan JR, Wang CF, Zhan D, Feng L, et al. Comparation on effects of Moutan Cortex, Paeoniae Rubra Radix, and Paeoniae Alba Radix on acute blood stasis model rats. Chinese Traditional and Herbal Drugs. 2016; 47: 2676-83. 
50. Wu CC, Guo XH, Liu X, Yang M, Wu W. Advances in modern pharmaceutical action of Moutan Cortex in recent five years. Chinese Journal of New Drugs. 2020; 29: 281-4.

51. Ding MJ, Deng XM, Meng J, Zhu QH, Wang SM, Liang SW. Simultaneous Assay of Three Kinds of Monoterpene Paeoniflorin Class and Paeonol in Moutan Cortex by Quantitative Analysis of Multi-components by Single Marker. Chinese Journal of Experimental Traditional Medical Formulae. 2014; 20: 80-5.

52. Ye JM, Deng T, Zhang JB. Influence of paeonol on expression of COX-2 and p27 in HT-29 cells. World journal of gastroenterology. 2009; 15: 4410-4.

53. Kyama CM, Overbergh L, Mihalyi A, Meuleman C, Mwenda JM, Mathieu C, et al. Endometrial and peritoneal expression of aromatase, cytokines, and adhesion factors in women with endometriosis. Fertility and sterility. 2008; 89: 301-10.

54. Jana S, Chatterjee K, Ray AK, DasMahapatra P, Swarnakar S. Regulation of Matrix Metalloproteinase-2 Activity by COX-2-PGE2-pAKT Axis Promotes Angiogenesis in Endometriosis. PloS one. 2016; 11: e0163540.

55. Lousse JC, Van Langendonckt A, González-Ramos R, Defrère S, Renkin E, Donnez J. Increased activation of nuclear factor-kappa B (NF-kappaB) in isolated peritoneal macrophages of patients with endometriosis. Fertility and sterility. 2008; 90: 217-20

56. Wang TS, Wang YH, Wu DL, Liu XG, Xuan ZH, Han L, et al. Effects of Different Extracts of Cortex Moutan on Hemorheology and Cytokines in Endometriosis Model Rats. Journal of Chinese Medicinal Materials. 2012; 35: 1649-52.

57. Zhu X, Fang ZH. New monoterpene glycosides from the root cortex of Paeonia suffruticosa and their potential anti-inflammatory activity. Natural product research. 2014; 28: 301-5.

58. Wang F, Wang L. Thinking and exploration on prescription-syndrome of Guizhi Fuling Pill. China Journal of Traditional Chinese Medicine and Pharmacy. 2016; 31: 2618-20.

59. Sun L, Li Y, Su Q, Tian S, Gao L. Effects of Guizhifuling pill combined with danazol and gestrinone capsule on immune inflammatory response and angiogenesis in patients with endometriosis. Hebei Medical Journal. 2019; 41: 1788-92.

60. Sun XL, Li YF, Su QQ, Tian SC, Gao LF. Effects of cassia fuling pill combined with danazol and progesterone capsule on immune inflammatory response and angiogenesis in patients with endometriosis. Hebei Medical Journal. 2019; 41: 1788-92.

61. Tao R, Yu CY. Effects of Guizhi Fuling Pill on MEK-2,p-ERK and VEGF Expression in Patients with Endometriosis. Journal of Liaoning University of Traditional Chinese Medicine. 2016; 18: 131-4.

62. You JH, Yan HR, Liu BH, Lian XY, Shen JZ, Yang SL, et al. Study on the law of traditional Chinese medicine for endometriosis. Journal of Chengdu University of Traditional Chinese Medicine. 2014; 37: 118-20.

63. Chang ZL. Effect of cassia twigi fuling pill on serum leptin, vascular endothelial growth factor, interleukin- 8 and ovarian function in patients with endometriosis. Modern Journal of Integrated Traditional Chinese and Western Medicine. 2016; 25: 3915-7.

64. Zhang YT, Zhao FR, Liu WW, Liu J, Fan HY. Clinical effect analysis of mifepristone combined with cassia branch tuckaing pill in the treatment of endometriosis. Progress in Modern Biomedicine. 2017; 17: 3703-6.

65. Olartecoechea B, Manero MG, Royo P, Aubà M. [Treatment for endometriosis]. Revista de medicina de la Universidad de Navarra. 2009.

66. Qian J. Clinical study on adding flavor of cassia twigi tuckaing pills in the treatment of endometriosis. Liaoning Journal of Traditional Chinese Medicine. 2000: 170 .

67. $\mathrm{Xu} \mathrm{JH}$, Zhang XD. Guizhi tuckaing pill was used to treat 50 cases of endometriosis. Henan Traditional Chinese Medicine. 2008: 21-2.

68. Feng Y, Feng GX. Thirty-nine cases of endometriosis were treated with acupuncture and medicine. Chinese Medicine Modern Distance Education of China. 2014; 12: 27-9.

69. Zhao JH, Zhu Y. Clinical application of cassia twig tuckaing pills. Henan Traditional Chinese Medicine. 2013; 33: 2071-2.

70. Li XL, Cui XP. Study on the treatment of endometriosis with cassia twigi tuckahoe pill. Modern Traditional Chinese Medicine. 2010; 30: 34-5.

71. Maheshwari A, Gurunath S, Fatima F, Bhattacharya S. Adenomyosis and subfertility: a systematic review of prevalence, diagnosis, treatment and fertility outcomes. Human reproduction update. 2012; 18: 374-92.

72. Vercellini P, Viganò $\mathrm{P}$, Somigliana E, Daguati R, Abbiati A, Fedele L. Adenomyosis: epidemiological factors. Best practice \& research Clinical obstetrics \& gynaecology. 2006; 20: 465-77.

73. Han FJ, Tian M. A review of the treatment of endometriosis by cassia twigi tuckahoe pill. Information on Traditional Chinese Medicine. 2015; 32: 124-6.

74. Liu F, Ju Y, Yang HY, Huang JL. Clinical efficacy of cassia twigi poria coiling pill combined with western medicine in the treatment of adenomyosis patients with secondary dysmenorrhea and its effect on serum il-6, PGE2 and ET. Shaanxi Journal of Traditional Chinese Medicine. 2016; 37: 1279-80.

75. Li YJ. Clinical study of radiofrequency ablation combined with traditional Chinese medicine in the treatment of adenomyosis. Maternal and Child Health Care of China. 2009; 24: 995-6.

76. Corson TW, Crews CM. Molecular understanding and modern application of traditional medicines: triumphs and trials. Cell. 2007; 130: 769-74.

77. Zhang $\mathrm{Y}, \mathrm{Wu} \mathrm{Z}$, Shu Y, Wang F, Cao W, Li W. A novel bioactive vaterite-containing tricalcium silicate bone cement by self hydration synthesis and its biological properties. Materials science \& engineering C, Materials for biological applications. 2017; 79: 23-9.

78. Fang LH, Wu XY, Luo SM. Curative effect of Sanjie Zhentong capsule combined with medroxyprogesterone acetate pill in treatment of endometriosis and the impacts on serum leptin,CA125, and estradiol. Maternal and Child Health Care of China. 2019; 34: 3916-9.

79. Xu J, Zhao W, Pan L, Zhang A, Chen $\mathrm{O}, \mathrm{Xu} \mathrm{K}$, et al. Peimine, a main active ingredient of Fritillaria, exhibits anti-inflammatory and pain suppression properties at the cellular level. Fitoterapia. 2016; 111: 1-6.

80. Su Z, Ke Z, Zhang X, Cao L, Wang Z, Xiao W. Molecular Mechanism of Sanjie Zhentong Capsule in Treatment of Endometriosis, Adenomyosis, Secondary Dysmenorrhea. Chinese Journal of Experimental Traditional Medical Formulae. 2019; 25: 165-72. 